\title{
Assessing the Global Distribution and Abundance of Marine Organisms
}

David L. Bradley

Pennsylzania State University - State College, Pennsylvania USA

\section{Background}

The Alfred P. Sloan Foundation and the Office of Naval Research sponsored a workshop in Monterey, California in January 1998, to discuss the value, timeliness, and feasibility of stimulating and organizing a period of intense, comprehensive oceanic observation whose purpose would be to assess and explain the global distribution and abundance of marine life, with emphasis on higher trophic levels. The meeting was organized by the Ocean Studies Board of the National Research Council and included a broad suite of ecologists, oceanographers, fisheries scientists, and modelers. A global assessment of marine life would aid in predicting the causes of ecosystem change and resulting consequences to fisheries, trophic structure, species interactions, and the population and community dynamics of marine systems. Several possible goals of a global assessment program were discussed including determine the biomass of the marine biota, especially the higher trophic levels, determine how this biomass is distributed spatially and by size and taxon, and investigate how these distributions are maintained or changed.

\section{The "Grand Challenge"}

In the 1960s, it was to put a man on the moon by 1970 . For the workshop, the similar Grand Challenge became "How many fish are in the sea?" However, determining how many fish are in the sea is a subset of the much broader major goal uniting all ecological research. That broader goal is to describe and understand the patterns of distribution and abundance of organisms and to predict the impact of change on those patterns. Participants redefined the original charge of the workshop in a way that could provide an overarching and unifying umbrella capable of encompassing the diverse interests of the biological community and which expressed a "Grand Challenge" in its most comprehensive and inspiring sense. Thus, the final "Grand Challenge" articulated by the workshop was to answer the question, "How much life can the ocean sustain?"

This challenge was made more concrete and attainable by focusing it primarily on higher trophic levels, including but not confined to economically important species. However, the distribution, abundance and productivity of lower trophic levels and the biological, chemical, and physical processes affecting ecosystem structure were also recognized as necessary to generate global estimates. The major questions thus became:

1. What is the biomass of the marine biota, especially higher trophic levels, on a global scale?

2. How is this biomass distributed spatially, by size, and by taxon?

3. How are these distributions maintained or changed?

\section{What (in addition to the sheer magnitude) makes the problem hard?}

Technological barriers prohibit the direct enumeration of most types of life in the ocean, except at very local scales. Sampling systems such as nets, and acoustical and optical instruments, which capture or count individual animals directly, can be deployed only over very small areas of the ocean at any Thus, the final "Grand Challenge" articulated by the zoorkshop was to answer the question,

"How much life can the ocean sustain?" one time using ships, moorings, or airplane overflights. Larger scale synoptic systems, such as satellites, are limited in the types of life they can detect, with phytoplankton pigments being the only biological parameter presently quantifiable with these techniques. Moreover, the patchy distributions of all organisms make it difficult to generalize local distributions and abundances to broader regional or global scales. 


\section{An assessment plan}

The most tractable approach to access the biomass and productivity of higher trophic levels at a regional scale was identified as a trophic dynamics approach. The abundance of higher trophic levels is constrained by the biomass at lower levels available for consumption, the efficiencies of trophic transfer at each link in the food web, and population parameters including rates of reproduction, growth, and mortality which alter population sizes and rates of trophic transfer.

Several components were seen as essential for an initial global assessment program. In general these components fell into three phases. During Phase I biogeographic zones would be defined and extensive review of existing data and models undertaken. The goal of the latter would be to clearly define major gaps in our knowledge. Development of important technology for population assessment would also begin in this phase. During Phase II, important gaps in the models would be filled by specific studies, both observational (population parameters of targeted functional groups measured in situ) and experimental (growth rates, reproductive rates etc.). In Phase III the models would be improved and tested during an iteration of Phase II.

\section{Conclusions}

Assessment of the biomass, abundance, and size distribution of open ocean taxa requires both significant advancements in new technology and better application of existing technologies, including acoustical, optical, genetic, and chemical approaches. The workshop participants felt that a global assessment program based on a trophic dynamics/modeling approach presented the prospect of significant scientific and societal benefits but would require the organization of a multiagency and multinational effort.

एव

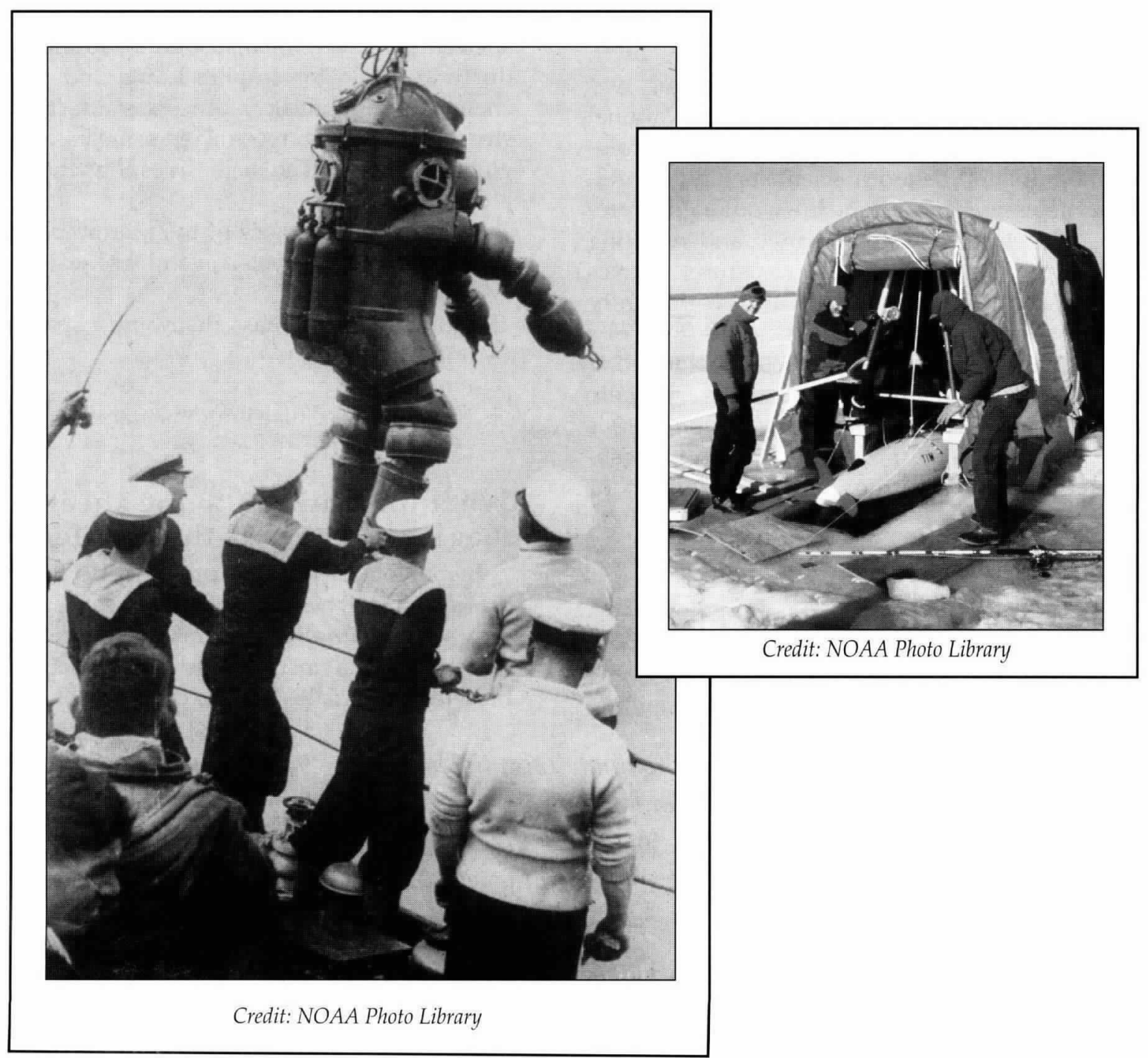

